\title{
Open letter: European Medicines Agency should remove barriers to access clinical trial data
}

In an open letter to Guido Rasi, director of the European Medicines Agency, the AllTrials campaign urges the EMA to revise its trial data policy or risk losing the trust of patients and healthcare professionals

\begin{abstract}
Ben Goldacre research fellow in epidemiology, London School of Hygiene and Tropical Medicine, Fiona Godlee editor in chief, The BMJ, Carl Heneghan director, Centre for Evidence based Medicine, Oxford, David Tovey editor in chief, Cochrane Library, Richard Lehman general practitioner, Oxfordshire, lain Chalmers coordinator, James Lind Initiative, Virginia Barbour medicine editorial director, PLOS, Tracey Brown managing director, Sense About Science
\end{abstract}

\section{Dear Professor Rasi}

Hundreds of supporters of the AllTrials campaign (alltrials.net) contributed last year to the European Medicines Agency's consultation on its draft policy "Publication and access to clinical-trial data." We welcomed the EMA's proposals, which would have seen clinical study reports published proactively and openly, in line with an agency policy that the information in those reports should not generally be considered commercially confidential. We are now concerned that the most recent draft of the policy (EMA/240810/2013), as shared with some stakeholders at meetings this month, introduces barriers that will make it all but useless to independent researchers.

We understand that there are four areas of particular concern:

- Clinical study reports (CSRs) will be available for viewing only on screen and cannot be saved, downloaded, printed, copied, annotated, or shared in any way. This will make it nearly impossible for researchers to usefully scrutinise the documents. CSRs often contain thousands of pages of complex information. Research teams need to be able to share information, and researchers have to be able to print or copy information such as outcome definitions to make valid comparisons across studies. Your policy on access to CSRs as initially proposed could have made a genuine contribution to medical research. It now risks becoming a superficial and practically useless gesture.

- The wording of the draft "Redaction Principles" policy is ambiguous, and where there is ambiguity there is likely to be excessive redaction. For example,

"statements/descriptions relating to objectives that are not supportive of a label claim and do not contribute to the overall benefit/risk evaluation" may be redacted. This would appear to suggest that any information on off-label uses of drugs will not be made available. While the agency's own use of these documents may be limited to a decision on one specific use of a treatment, in everyday clinical practice these same treatments are routinely and legally used by clinicians outside their marketing authorisation. This prescribing is based on published evidence that may be particularly vulnerable to dissemination bias, since evidence on off-label uses is frequently excluded from even voluntary codes of practice.

- The "Redaction Principles" policy asks sponsors to submit two versions of a CSR from each trial: a standard version (CSR(a)) and a redacted version (CSR(b)). While this might be driven by a desire to lower the costs of transparency for the agency, it puts primary responsibility for redacting in the hands of sponsors. How close $\operatorname{CSR}(\mathrm{a})$ is to $\operatorname{CSR}(\mathrm{b})$, and which parts are missing, may never be known to researchers granted access to CSR(b). Will there be an audit to explore whether redactions are proportionate or risk patient safety, and will there be penalties for excessive redactions?

- The Terms of Use contract contains clauses that seem to prioritise trial sponsors' legal rights over researchers' need to scrutinise and use information from CSRs to improve the evidence base for patient care. These supposed rights given to sponsors have never been established in law and are currently being debated in court cases in which the agency is involved. Of greater concern, researchers will be asked to agree to a clause that allows trial sponsors to take direct legal action against the researcher for possible violation of the terms of use. Individual researchers are therefore being made vulnerable to protracted legal battles with large companies for infractions to these cumbersome 
rules. Since the rules themselves are poorly specified, these cases are likely to involve lengthy legal discussion. They will therefore introduce a new and unpredictable risk of high legal costs into routine academic work, effectively chilling researchers' ability to use information relevant to patient care.

You have probably heard from some quarters that information from clinical trials is commercially confidential. We have heard one drug company lawyer (from AbbVie) even go as far as claiming that information on adverse events should be kept as trade secrets. However, some companies and most academic institutes are throwing off the culture of secrecy and moving forward. The recently agreed European Clinical Trials Directive will enshrine the policy that information in CSRs is not generally commercially confidential in law.

There is no good reason to introduce barriers to access CSRs, as the agency's draft policy does. We know you agree that allowing independent researchers to be free to scrutinise CSRs will have huge benefits for patients, doctors, pharmacists, regulators, and researchers and that these will inform treatment decisions now and decisions about future research.

AllTrials is a campaign for all clinical trials to be registered and results reported. It is supported by more than 78000 people and
470 organisations worldwide. The numbers of European citizens and organisations supporting it are growing every day-and will continue to grow and press for change. We ask you to revise the policy to reflect your earlier support for the public interest in better medicine. We are entering a new era of medicine, one where medical practice is based on evidence that is openly available and critically appraised rather than on edicts and eminence. If the EMA allows its new policy to be based on out of date attitudes it risks losing the trust of patients, policy makers, and healthcare professionals. We urge you to revise your policy to reflect your earlier support for the public interest in better medicine.

Acknowledgment: We thank T Jefferson, P Doshi, T Lemmens, and B Wieseler for their comments on the draft EMA policy.

Provenance and peer review: Not commissioned; not peer reviewed.

Competing interests: All authors are members of the steering committee of the AllTrials campaign (alltrials.net).

For more on The BMJs open data campaign go to bmj.com/open-data.

Cite this as: BMJ 2014;348:g3768

(c) BMJ Publishing Group Ltd 2014 